\title{
APATITIC CRYSTAL GROWTH ON HYDROXYAPATITE AND SOME OXIDE MATERIALS COATED ON FERROELECTRIC CERAMICS
}

\author{
Eiko Yonehara, Kimihiro Yamashita, Takao Umegaki \\ Department of Industrial Chemistry, Tokyo Metropolitan University \\ Hachioji, Tokyo 192-03, Japan
}

\begin{abstract}
Electric poling was found to enhance crystal growth on hydroxyapatite by the effect of $\mathrm{BaTiO}_{3}$ The enhancement was observed on the negatively polarized surface, while the crystal growth was restricted on the positively poled surface. Based on these results, the effect was attributed to the condensation of $\mathrm{Ca}^{2+}$ ions due to the electrostatic attraction of aligned dipole moments of $\mathrm{BaTiO}_{3}$
\end{abstract}

\section{INTRODUCTION}

Not only the unparalleled bioactivity but also the electric properties of hydroxyapatite (HAp) are partly attributed to its lattice $\mathrm{OH}^{-}$ions, which are surrounded with $\mathrm{Ca}^{2+}$ in the HAp structure and are reportedly stable up to ca. $1000 \mathrm{~K}$ in air. The dielectric measurements were carried out at first for the understanding of the bioactive characteristics of HAp. As a result, HAp ceramics were proved to exhibit dielectric characteristics below ca. $800 \mathrm{~K}$. The later studies by the thermally stimulated current method showed that HAp ceramics were polarizable above the monoclinic-hexagonal phase transition temperature $(\sim 500 \mathrm{~K})$ due to the reorientation of the lattice $\mathrm{OH}^{-}$ions along the c-axis. Taking the polarizability into account, we conducted the study on the poling effect on the crystal growth on HAp ceramics. Very recently, we observed the remarkable effect of electric poling on crystal growth. $(1,2)$

$\mathrm{BaTiO}_{3}$ is well-known as a material with a high dielectric constant. $\mathrm{BaTiO}_{3}$ has spontaneous polarization, which can be oriented by an external electric field. This report shows how poled $\mathrm{BaTiO}_{3}$ has an influence on apatitic crystal growth.

\section{METHODS AND MATERIALS}

Laminated films of $\mathrm{BaTiO}_{3}$ and HAp were prepared by electrophoretic deposition (EPD) (Fig.1). BaTiO3 powders (Kanto chemicals) and HAp powders (Taihei chemicals) were used. After electrophoretic deposition, the specimens were sintered at $1473 \mathrm{~K}$. The crashed powders of sintered specimens were analyzed by X-ray diffraction (XRD). The as-sintered ceramic specimens, which were sandwiched between Pt plates, were poled using a dc field of $1200 \mathrm{~V} / \mathrm{cm}$ at $473 \mathrm{~K}$ for $5 \mathrm{~min}$. The poled specimens were quenched to room temperature, then immersed in a simulated body fluid (SBF) for $1 \mathrm{~h} \sim 48 \mathrm{~h}$. The washed and dried specimens were subjected to scanning electron microscopy (SEM).

\section{RESULTS AND DISCUSSION}

Figure 1 shows the X-ray diffraction pattern of the sintered specimen, indicating that HAp and $\mathrm{BaTiO}_{3}$ remained after sintering at $1473 \mathrm{~K}$.

Figure 2 shows the HAp surface before immersion. It shows the HAp-coated surface. 


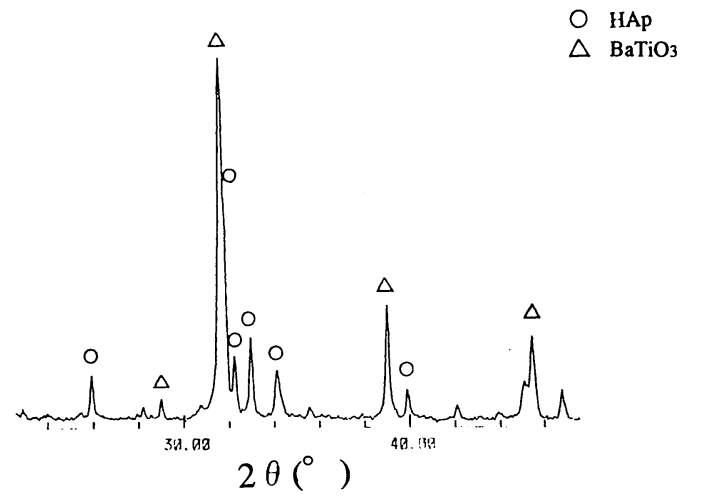

Fig.1 X-ray diffraction pattern of the $\mathrm{HAp}$-coated $\mathrm{BaTiO}_{3}$ specimen sintered at $1473 \mathrm{~K}$.

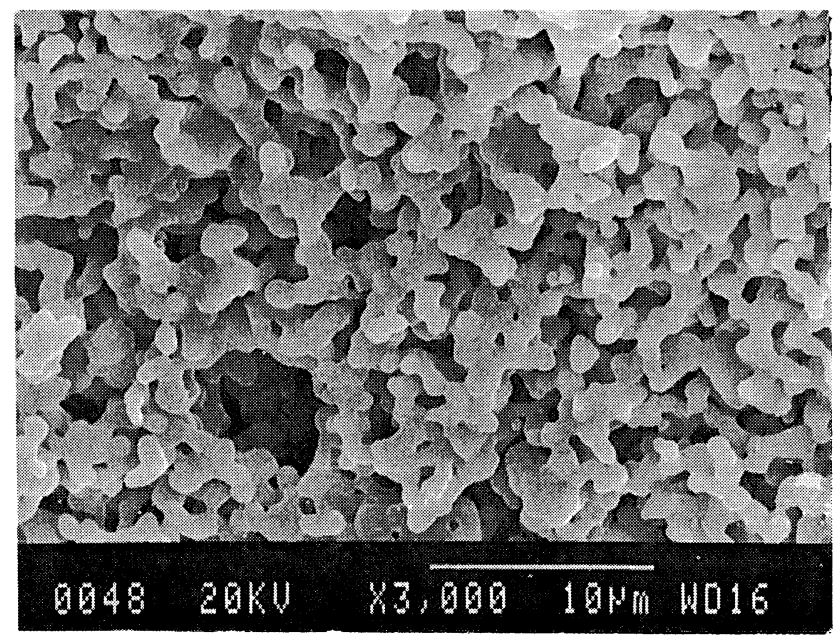

Fig.2 HAp-coated surface before immersion.

Figure 3 shows the SEM photographs of the negatively polarized HAp surfaces of the poled specimens after immersion in SBF. It was observed that crystal growth took place even in $1 \mathrm{~h}$, and the crystal grew with time. Therefore it was shown that negative charges accelerate crystal growth on HAp (Fig.4). On the other hand, Fig.5 shows the SEM photographs of the positively polarized HAp surfaces of the specimens after immersion in SBF, and no crystals were grown on the positively charged surface even in $48 \mathrm{~h}$. The crystal growth was observed after 2 week-immersion. Therefore the poling effect of preventing crystal growth was found to be maintained for a considerable period.

Previous study has shown that the crystal growth occurs on the negatively polarized surfaces of the poled HAp in SBF within a few hours. Furthermore, this study shows that the crystal growth occurs within only $1 \mathrm{~h}$. by the effect of poled $\mathrm{BaTiO}_{3}$. 


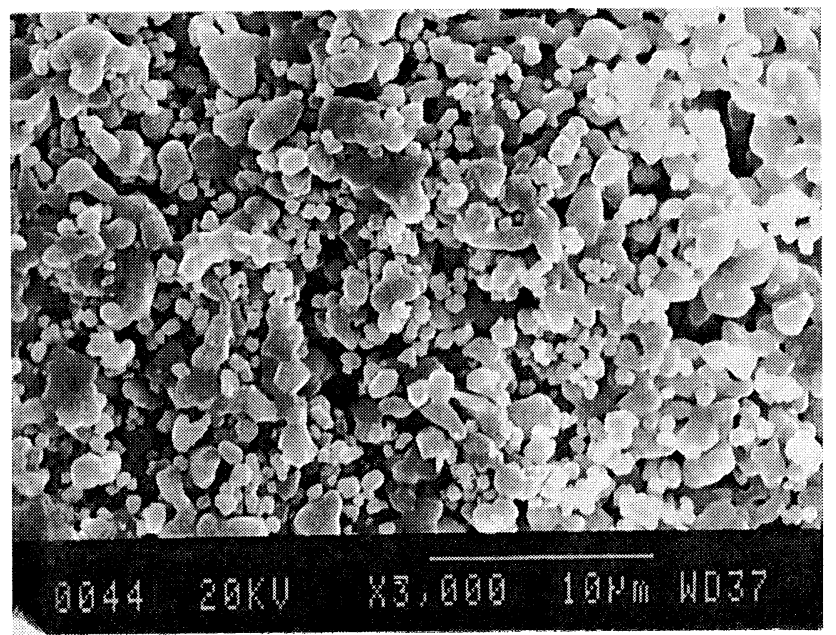

(A) $1 \mathrm{~h}$.

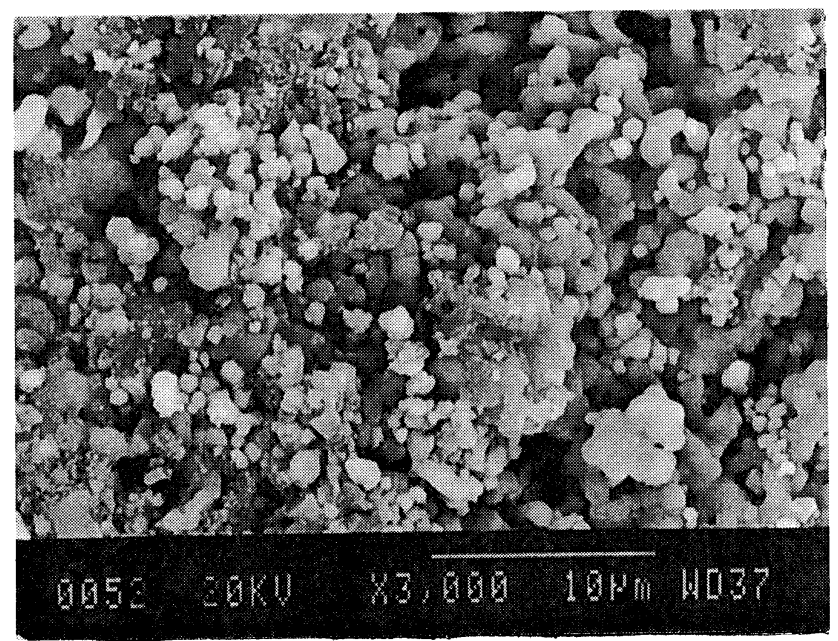

(B) $3 \mathrm{~h}$.

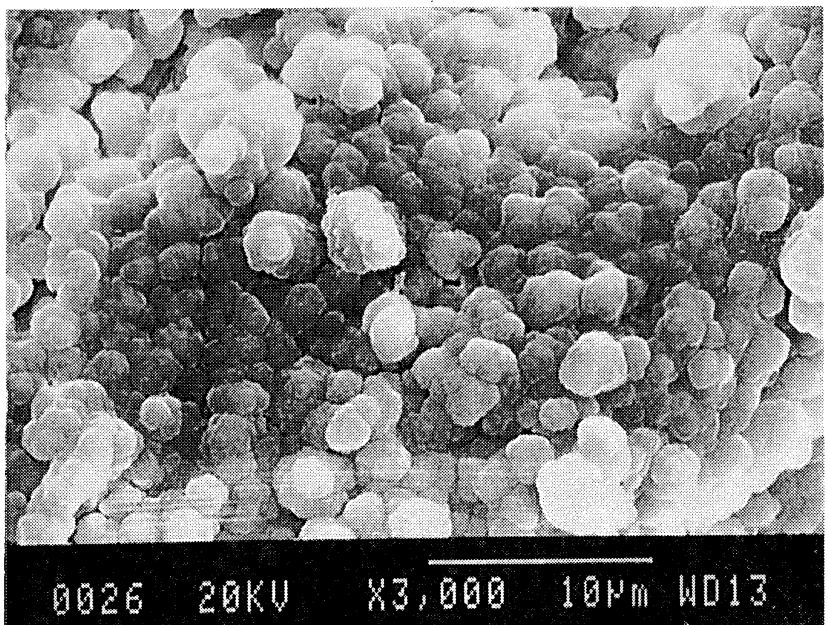

(C) $12 \mathrm{~h}$.

Fig.3 Change of negatively polarized HAp surfaces of the poled specimens after immersion

in $\mathrm{SBF}$. 


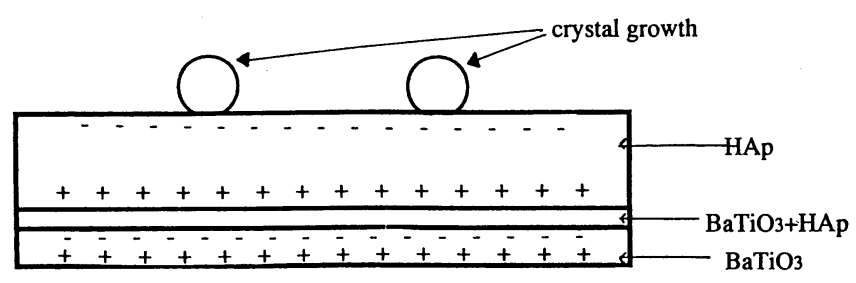

Fig.4 Apatitic crystal growth model on HAp

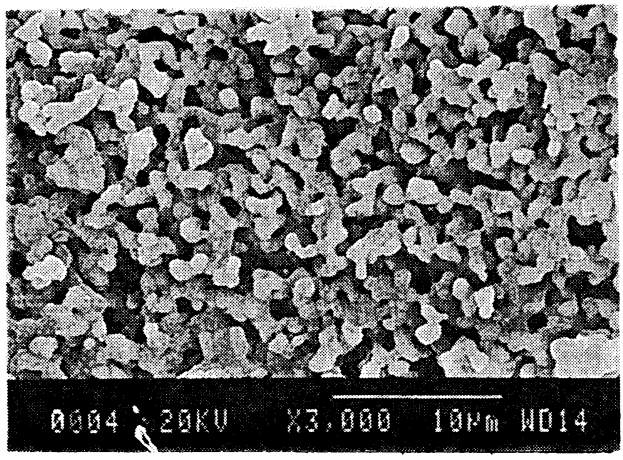

(A) $48 \mathrm{~h}$.

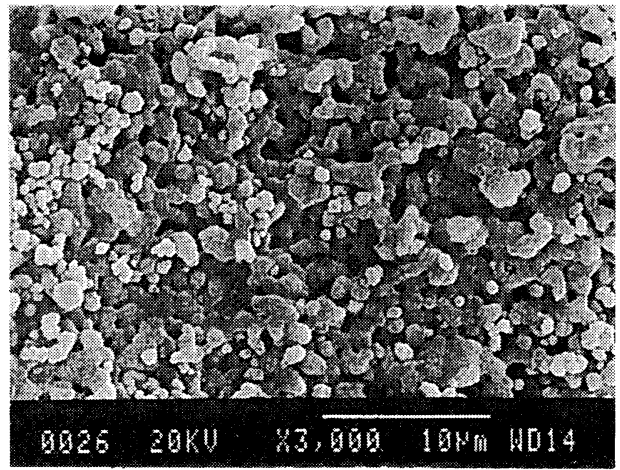

(B) 2 weeks

Fig.5 Positively polarized HAp surfaces of the poled specimens after immersion in SBF.

\section{SUMMARY}

Crystal growth on HAp was enhanced or restricted by changing the poling direction of BaTiO3.

\section{ACKNOWLEDGEMENT}

This work was partly supported by the Ministry of Education, Sports, Culture and Science of Japan. One of the authors (K.Y) would like to express his thank to Kumagaya Science Foundation for the support of his study on "Development of self-curable biomaterials" .

\section{REFERENCES}

(1) Kimihiro Yamashita, Noriyuki Oikawa, Takao Umegaki, Proc. 2nd Pac Rim Cont. Ceramics (in press) (1996).

(2) Kimihiro Yamashita, Noriyuki Oikawa, Takao Umegaki, Mat. Chem., (in press). 